\title{
Nível de estresse de gestores de unidades básicas de saúde
}

\author{
Stress level of managers of basic health units
}

Nivel de estrés de gerentes de unidades básicas de salud

Camila Cruz Damascenoำ Cláudia Cristiane Filgueira Martins Rodrigues ${ }^{1}$, Rosires Magali Bezerra de Barros ${ }^{1}$, Pétala Tuani Candido de Oliveira Salvador ${ }^{1 *}$.

\section{RESUMO}

Objetivo: Identificar o nível de estresse de gestores de Unidades Básicas de Saúde de um município do Nordeste do Brasil. Métodos: Trata-se de estudo do tipo descritivo, com abordagem quantitativa. Os campos de pesquisa foram as Unidades Básicas de Saúde (UBS) e as Unidades de Saúde da Família (USF) dos cinco Distritos Sanitários (Leste, Oeste, Sul, Norte I e Norte II) de Natal-RN, Brasil, no primeiro semestre de 2018, de modo que a amostra final foi de 44 gestores. Os resultados foram digitados em planilha eletrônica e a análise dos dados foi realizada por meio da estatística descritiva simples e análise inferencial. Resultados: participaram do estudo 44 gestores: a maioria do sexo feminino, com média de idade de 49,41 anos ( $D P=8,92$ ). Com relação à fase do estresse em que os gestores estavam, observou-se que 17 (38,6\%) estavam na Fase de Resistência; 2 (4,5\%) na fase de Quase Exaustão; e 10 (22,7\%) encontravam-se na fase de exaustão. Conclusão: evidenciou-se que a maioria dos gestores das Unidades Básicas de Saúde estudadas apresentam estresse, e uma parcela desses já se insere na fase de Exaustão, que é quando o indivíduo começa a ficar suscetível ao surgimento de doenças.

Palavras-chave: Estresse psicológico, Estresse fisiológico, Esgotamento profissional, Administração de serviços de saúde, Gestão em saúde.

\section{ABSTRACT}

Objective: To identify the level of stress of the managers of the Basic Health Units of a municipality in northeastern Brazil. Methods: This is a descriptive study, with a quantitative approach. The research fields were the Basic Health Units (UBS) and the Family Health Units (USF) of the five Health Districts (East, West, South, North I and North II) of Natal-RN, Brazil, in the first semester 2018, so that the final sample was 44 managers. The results were entered into an electronic spreadsheet and data analysis was performed using simple descriptive statistics and inferential analysis. Results: 44 managers participated in the study: the majority were female, with a mean age of 49.41 years $(S D=8.92)$. Regarding the stress phase that the managers were in, it was observed that $17(38.6 \%)$ were in the Resistance Phase; 2 (4.5\%) in the Near Exhaustion phase; and 10 (22.7\%) were in the exhaustion phase. Conclusion: It was evidenced that the majority of the managers of the Basic Health Units present stress, and a part of them is already inserted in the Exhaustion phase, which is when the individual starts to be susceptible to the appearance of diseases.

Keywords: Psychological stress, Physiological stress, Burnout, Health services administration, Health management.

\section{RESUMEN}

Objetivo: Identificar el nivel de estrés de los gerentes de las Unidades Básicas de Salud de un municipio en el noreste de Brasil. Métodos: Este es un estudio descriptivo, con un enfoque cuantitativo. Los campos de investigación fueron las Unidades Básicas de Salud (UBS) y las Unidades de Salud Familiar (USF) de los cinco Distritos de Salud (Este, Oeste, Sur, Norte I y Norte II) de Natal-RN, Brasil, en el primer semestre. 2018. El análisis de datos se realizó mediante estadísticas descriptivas simples y análisis inferencial. Resultados: 44 gerentes participaron en el estudio: la mayoría eran mujeres, con una edad media de 49,41 años $(\mathrm{DE}=$

1Universidade Federal do Rio Grande do Norte, Natal - RN. *E-mail: petalatuani@hotmail.com

SUBMETIDO EM: 8/2020

ACEITO EM: 9/2020

PUBLICADO EM: 10/2020 
8,92). Con respecto a la fase de estrés en la que se encontraban los gerentes, se observó que 17 (38,6\%) estaban en la fase de resistencia; $2(4.5 \%)$ en la fase de agotamiento cercano; y 10 (22.7\%) estaban en la fase de agotamiento. Conclusión: Se evidenció que la mayoría de los gerentes de las Unidades Básicas de Salud presentan estrés, y una parte de ellos ya está insertada en la fase de Agotamiento, que es cuando el individuo comienza a ser susceptible a la aparición de enfermedades.

Palabras clave: Estrés psicológico, Estrés fisiológico, Agotamiento, Administración de servicios de salud, Gestión de la salud.

\section{INTRODUÇÃO}

O estresse está associado a um conjunto de reações psicofisiológicas e comportamentais complexas. Esse transtorno surge como um mecanismo de defesa, gerado pelo organismo com o objetivo de manter seu equilíbrio frente situações de difícil adaptação. Caso esse evento estressor a que o indivíduo está submetido seja excessivo, gera a quebra da sua homeostase interna, dificultando a manutenção do equilíbrio, gerando o adoecimento (GEREMIAS LM, et al., 2017).

$\mathrm{Na}$ área da saúde, o termo foi inicialmente utilizado pelo médico Hans Seyle, que o conceituou como um resultado inespecífico de qualquer demanda sobre o corpo, seja de efeito mental ou somático, em que o estressor é todo agente de demanda que provoca uma reação de estresse, seja de natureza física, mental ou emocional (ABREU KL, et al., 2002).

O estresse ocupacional, por sua vez, é aquele que está ligado à profissionalização e ao desenvolvimento da sociedade, ou seja, está a cargo do trabalhador administrar a responsabilidade profissional e saber lidar com as situações conflituosas geradas pela sociedade e pelas pessoas (LENTINE EC, et al., 2003).

A exposição repetida e prolongada a eventos estressores conduz o corpo físico a vivenciar o estresse, que se subdivide em quatro fases. A fase de alerta é considerada a fase positiva, em que o ser humano se energiza por meio da produção de adrenalina, a sobrevivência é preservada e uma sensação de plenitude é frequentemente alcançada; na segunda fase, de resistência, o organismo usa toda a sua energia para se equilibrar, tentando obter sua homeostase interna (LIPP ME, 1996).

A terceira fase é denominada de quase exaustão, por se encontrar entre a fase de resistência e a de exaustão. Nesta fase, as defesas do organismo começam a ceder e ele já não consegue resistir às tensões e restabelecer a homeostase. Caracteriza-se por um enfraquecimento da pessoa que não consegue mais se adaptar ou resistir ao estressor e, assim, as doenças começam a surgir, embora, não sejam tão graves, como na fase de exaustão (LIPP ME, 1996).

A quarta fase, a exaustão, acontece se o estressor é contínuo e a pessoa não possui estratégias para lidar com o estresse, de modo que o organismo exaure sua reserva de energia adaptativa e doenças sérias aparecem. Há uma queda na imunidade e o surgimento da maioria das doenças (LIPP ME, 1996).

No que se refere ao estresse ocupacional que é relacionado ao ambiente de trabalho, Coutinho DA e Santos JW (2010) apontam falta de autonomia como fator de estresse dentro das organizações, como também: excesso de tarefas, pressão, condições de trabalho. Mediante isso, o indivíduo fica susceptível a adquirir problemas de saúde físicos e/ou emocionais.

Dentre os ambientes de trabalho, por sua vez, o serviço de saúde é visto como ambiente repleto de estressores (ESTEVES GGL, et al., 2019). Entre os profissionais de saúde, os gestores encontram-se propensos ao estresse e suas consequências, por se tratar de um cargo em que os profissionais lidam diariamente com situações em que os relacionamentos interpessoais se tornam conturbados, tanto com os membros da equipe multiprofissional como com os usuários (MAFFIA LN e PEREIRA LZ, 2014).

A responsabilidade em exercer as funções pertencentes aos serviços de saúde, assim como também o atual cenário da saúde pública no Brasil, que apresenta problemas que os gestores também precisam lidar como a falta de recursos para uma alta necessidade, corrobora para a construção de um ambiente de trabalho tenso e com alto nível de insatisfação e estressores (MAFFIA LN e PEREIRA LZ, 2014). 
Dentre os ambientes de saúde de atuação dos gestores, destacam-se as Unidades Básicas de Saúde, em que está instalado o primeiro nível de atenção à saúde, também nomeado de atenção primária. A Atenção Primária à Saúde (APS) é o nível do sistema de serviços de saúde que oferece a entrada para todas as novas necessidades e problemas, fornece atenção sobre a pessoa (não direcionada para enfermidade) no decorrer do tempo, fornece atenção para todas as condições, exceto as muito incomuns e raras, e coordena ou integra a atenção fornecida em outro lugar por terceiros (STARFIELD B, 2002).

Diante desse contexto, a APS é considerada como a porta de entrada do serviço público de saúde, consequentemente possui uma grande demanda, cenário em que o gestor se torna alvo de sobrecarga devido às atividades relacionadas à gerência e às partes burocráticas, tornando-o propenso a desenvolver o estresse (CAMPOS EP, et al., 2010).

Dessa forma, este estudo teve como questões norteadoras: Os gestores das Unidades Básicas de Saúde possuem estresse? Se sim, quais são suas fases? E quais os sinais e sintomas de maior incidência?

Objetiva-se, portanto, identificar o nível de estresse dos gestores das Unidades Básicas de Saúde de um município do Nordeste do Brasil.

\section{MÉTODOS}

O estudo é do tipo descritivo, com abordagem quantitativa. Os campos de pesquisa foram as Unidades Básicas de Saúde (UBS) e as Unidades de Saúde da Família (USF) dos Distritos Sanitários de um município do Rio Grande do Norte, Brasil, os quais prestam assistência básica de saúde à população da capital potiguar (Tabela 1).

Tabela 1 - Campo de pesquisa. Natal/RN, 2020.

\begin{tabular}{ccc}
\hline Distrito & № de Unidades & № de Gestores Entrevistados - n (\%) \\
\hline 1 & 8 & $6(13,6 \%)$ \\
2 & 12 & $11(25,0 \%)$ \\
3 & 8 & $8(18,2 \%)$ \\
4 & 10 & $9(20,5 \%)$ \\
5 & 11 & $10(22,7 \%)$ \\
\hline Total & 49 & $44(100 \%)$ \\
\hline
\end{tabular}

Fonte: DAMASCENO CC, et al., 2020.

A população foi constituída por 49 gestores. Os critérios de inclusão utilizados foram: Ser Gestor ou Administrador da Unidade e ter mais de seis meses atuando como gestor, que é o tempo considerado adequado para inserção no cotidiano laboral do serviço. A amostra final foi de 44 gestores - dois foram excluídos por atuarem há menos de seis meses e três por não ser possível agendamento da entrevista, mesmo após três tentativas.

A coleta de dados ocorreu no primeiro semestre do ano 2018 e se deu através do agendamento de horário e dia com o próprio gestor em sua respectiva UBS de atuação. Foram utilizados como instrumentos de coleta: formulário de caracterização, com perguntas acerca da formação do gestor, sua idade, quanto tempo ele atua no cargo, qual seu cargo atual na unidade, se possui algum curso de gestão em saúde e qual função exercia antes do cargo de gestor; e o inventário de sinais e sintomas de Lipp (ISSL).

O ISSL é composto de 56 itens de autopreenchimento, sendo 37 de natureza somática física e 19 de natureza psicológica. $O$ instrumento constitui-se de três quadros: o primeiro refere-se aos sintomas apresentados nas últimas 24 horas - fase de alerta; o segundo enquadra os sintomas experimentados na última semana - fases de resistência e quase exaustão; e o terceiro identifica os sintomas apresentados no último mês - fase de exaustão (LIPP ME, 1996). Os resultados foram digitados em planilha eletrônica e a análise dos dados foi realizada por meio de estatística descritiva simples e análises inferenciais. 
A pesquisa seguiu as normas e diretrizes propostas pela resolução 466/12 do Conselho Nacional de Saúde (CNS), que regulamenta as pesquisas envolvendo seres humanos. Foi submetida à apreciação do Comitê de Ética e Pesquisa, sendo aprovado a partir do Parecer Consubstanciado ํㅜ‥400.919, de 27 de novembro de 2017, CAAE no 76797517.4.0000.5537.

\section{RESULTADOS}

Participaram do estudo 44 gestores: a maioria $(28 ; 63,6 \%)$ era do sexo feminino, com média de idade de 49,41 anos (DP = 8,92), sendo a idade mínima de 30 anos e a máxima de 62 anos. Quanto ao nível de formação, 8 (18,2\%) gestores possuíam apenas nível médio, 23 (52,3\%) apresentavam nível superior, sendo este o maior percentual, e $13(29,5 \%)$ detinham nível de especialização. Com relação à existência de capacitação em gestão, identificou-se que $14(31,8 \%)$ eram graduados em cursos na área de administração e $30(68,2 \%)$ não possuíam nenhuma capacitação na área.

Com relação ao tempo de atuação como gestor, o mínimo foi de 7 meses e o máximo de 28 anos. Já no que se refere ao Tempo de Atuação na Unidade (atuação como gestor e função desempenhada antes da gestão), os gestores possuíam um mínimo de 2 meses e um máximo de 29 anos de atuação, com média de 7 anos (DP $=7$ ).

A partir dos resultados do ISSL, foi possível detectar que 29 (65,9\%) gestores apresentavam algum nível de estresse. Com relação à fase do estresse em que os gestores estavam, observou-se que $17(38,6 \%)$ gestores estavam na Fase de Resistência, caracterizada pelo aumento de cortisol, onde o corpo utiliza de toda sua energia para manter-se em equilíbrio; 2 (4,5\%) indivíduos estavam na fase de Quase Exaustão; e $10(22,7 \%)$ encontravam-se na fase de Exaustão, dado que se mostra preocupante, pois é nessa fase que surgem as doenças mais graves, bem como o desenvolvimento de outras patologias associadas.

Foi possível, ainda, identificar os sinais e sintomas de ordem física e psicológica que os gestores autorreferiram possuir, sendo identificada predominância de ordem física $(34 ; 77,3 \%)$, o que já denota um processo de adoecimento em curso (Tabela 2).

Tabela 2 - Presença dos sintomas físicos e psicológicos.

Natal/RN, 2020.

\begin{tabular}{lcc}
\hline Sintomas predominantes & $\mathbf{n}$ & $\%$ \\
\hline Sintomas físicos & 34 & 77,3 \\
Sintomas psicológicos & 9 & 20,5 \\
Sintomas físicos e psicológicos & 1 & 2,3 \\
\hline Total & 44 & 100 \\
\hline
\end{tabular}

Fonte: DAMASCENO CC, et al., 2020.

Sendo assim, esses sintomas podem ser enquadrados como reflexo das fases de estresse, já citadas, uma vez que se relacionam à presença e ou desenvolvimento de alguma morbidade que esteja a se desenvolver no organismo do indivíduo.

Dentre os sintomas físicos do estresse, os que mais se destacaram foram: insônia ( $31 ; 6,9 \%)$; tensão muscular (29; 4,5\%); problemas com a memória (28; 6,3\%); e sensação de desgaste físico constante (28; 6,3\%) (Tabela 3).

Dentre os sintomas psicológicos, destacou-se a angústia ou ansiedade diária (24; 10,4\%) (Tabela 4). 
Tabela 3 - Sintomas físicos autorreferidos pelos gestores. Natal/RN, 2020.

\begin{tabular}{|c|c|c|}
\hline Sintomas & $\mathbf{n}$ & $\%^{*}$ \\
\hline Insônia & 31 & 6,9 \\
\hline Tensão muscular (dor muscular) & 29 & 6,4 \\
\hline Problemas com memória & 28 & 6,2 \\
\hline Sensação de desgaste físico constante & 28 & 6,2 \\
\hline Dificuldade para dormir & 27 & 6,0 \\
\hline Cansaço constante & 23 & 5,1 \\
\hline Mudança de apetite & 19 & 4,2 \\
\hline Hipertensão arterial (pressão alta) & 18 & 4,0 \\
\hline Tontura & 15 & 3,3 \\
\hline Nó ou dor no estômago & 14 & 3,1 \\
\hline Hipertensão confirmada & 13 & 2,9 \\
\hline Boca seca & 13 & 2,9 \\
\hline Mal-estar generalizado & 12 & 2,6 \\
\hline Taquicardia (batimento acelerado) & 12 & 2,6 \\
\hline Aparecimento de problemas dermatológicos (pele) & 12 & 2,6 \\
\hline Formigamento nas extremidades (mãos e pés) & 11 & 2,4 \\
\hline Formigamento & 11 & 2,4 \\
\hline Mudança extrema de apetite & 10 & 2,2 \\
\hline Aparecimento gastrite prolongada (queimação, azia) & 9 & 2,01 \\
\hline Diarreias frequentes & 9 & 2,01 \\
\hline Hipertensão súbita e passageira & 9 & 2,01 \\
\hline Problemas dermatológicos prolongados & 8 & 1,8 \\
\hline Tontura frequente & 8 & 1,8 \\
\hline Respiração ofegante & 8 & 1,8 \\
\hline Aumento de sudorese & 8 & 1,8 \\
\hline Dificuldades sexuais & 7 & 1,6 \\
\hline Mãos/pés frios & 6 & 1,3 \\
\hline Aperto na mandíbula/ranger de dentes & 6 & 1,3 \\
\hline Diarreia passageira & 6 & 1,3 \\
\hline Impossibilidade de Trabalhar & 3 & 0,67 \\
\hline Úlcera & 0 & 0 \\
\hline
\end{tabular}

Legenda: *a soma das porcentagens supera $100 \%$ pois os gestores assinalaram mais de um sintoma.

Fonte: DAMASCENO CC, et al., 2020. 
Tabela 4 - Sintomas psicológicos autorreferidos pelos gestores. Natal/RN, 2020.

\begin{tabular}{lcc}
\hline Sintomas & $\mathbf{n}$ & $\%^{*}$ \\
\hline Angustia ou ansiedade diária & 24 & 10,4 \\
Cansaço excessivo & 21 & 9,1 \\
Vontade súbita de iniciar novos projetos & 21 & 9,1 \\
Vontade de fugir de tudo & 18 & 7,8 \\
Irritabilidade excessiva & 17 & 7,3 \\
Pensamento constante sobre um só assunto & 14 & 6,1 \\
Irritabilidade excessiva & 14 & 6,1 \\
Hipersensibilidade emotiva & 13 & 5,6 \\
Sensação de incompetência em todas as áreas & 10 & 4,3 \\
Aumento súbito de motivação & 10 & 4,3 \\
Entusiasmo súbito & 9 & 3,9 \\
Irritabilidade sem causa aparente & 9 & 3,9 \\
Dúvida quanto a si próprio & 9 & 3,9 \\
Pesadelos & 8 & 3,5 \\
Diminuição da libido (desejo sexual) & 8 & 3,5 \\
Perda do senso de humor & 8 & 3,5 \\
Apatia & 6 & 2,6 \\
\hline
\end{tabular}

Legenda: *a soma das porcentagens supera $100 \%$ pois os gestores assinalaram mais de um sintoma.

Fonte: DAMASCENO CC, et al., 2020.

A partir de análises inferenciais, foi possível verificar a associação de características do perfil dos entrevistados com a presença de estresse (Tabelas 5 e 6).

Tabela 5 - Relação entre idade e tempo de atuação com presença de estresse. Natal/RN, 2020.

\begin{tabular}{cccc}
\hline Variável & Com estresse & Sem estresse & $\mathbf{p}^{*}$ \\
\hline Idade & $\mathbf{X}$ (DP) & $\mathbf{X}$ (DP) & \multicolumn{1}{c}{ (D) } \\
\hline Tempo de atuação como Gestor (meses) & $49,0(9,2)$ & $50,2(8,5)$ & 0,670 \\
Tempo de atuação na Unidade (meses) & $112,24(78,9)$ & $136,3(99,2)$ & 0,423 \\
& $93,4(86,6)$ & $64,3(89,6)$ & 0,311 \\
\hline
\end{tabular}

Legenda: *Teste t de Student.Fonte: DAMASCENO CC, et al., 2020.

Sendo assim, pode-se observar que não houve relação significativa entre as variáveis idades e tempo de atuação com a presença do estresse, dado que nenhum resultado foi de $p<0,05$. Apesar disso, observa-se que os gestores com estresse apresentavam média de idade e de tempo de atuação como gestores menores quando comparados aos gestores sem estresse; em contrapartida, atuavam na unidade há mais tempo que os gestores sem estresse.

Tabela 6 - Relação entre variáveis categóricas e presença de estresse. Natal/RN, 2018.

\begin{tabular}{lccc}
\hline Variável & $\begin{array}{c}\text { Com estresse } \\
\mathbf{n}(\%)\end{array}$ & $\begin{array}{c}\text { Sem estresse } \\
\mathbf{n}(\%)\end{array}$ & $\mathbf{p}^{*}$ \\
\hline Sexo & & & \\
\hline Masculino & $11(38,0 \%)$ & $5(33,3 \%)$ & 0,516 \\
Feminino & $18(62,0 \%)$ & $10(66,7 \%)$ & \\
\hline Capacitação em Gestão & & & \\
\hline Não & $3(10,4 \%)$ & $8(53,3 \%)$ & 0,003 \\
Sim & $26(89,6 \%)$ & $7(46,7 \%)$ & \\
\hline Titulação & & & \\
\hline Nível médio & $6(20,6 \%)$ & $2(13,3 \%)$ & 0,438 \\
Nível superior & $23(79,4 \%)$ & $13(86,7 \%)$ & \\
\hline Graduação na área da Gestão e/ou Saúde & & & \\
\hline Não & $21(72,4 \%)$ & $9(60,0 \%)$ & 0,307 \\
Sim & $8(27,6 \%)$ & $6(40,0 \%)$ & 0,300 \\
\hline
\end{tabular}

Legenda: *Qui-quadrado. Fonte: DAMASCENO CC, et al., 2020. 
Assim, verificou-se relação significativa $(p=0,003)$ entre a presença de Capacitação em Gestão e a presença de estresse, o que pode indicar que o maior conhecimento acerca do processo de trabalho nas organizações de saúde gera maior responsabilidade, sendo assim, uma vertente importante para o desenvolvimento de fatores estressores.

\section{DISCUSSÃO}

Os dados obtidos permitem afirmar que a amostra foi composta prioritariamente pelo sexo feminino $(63,6 \%)$. Levando em conta os estudos já realizados com levantamento dos gêneros no trabalho do setor da saúde, como o artigo "A Força de Trabalho do Setor de Saúde no Brasil: Focalizando a Feminização", feito por cinco pesquisadoras da Fundação Oswaldo Cruz (Fiocruz), é visível a assimetria entre trabalhadores homens e mulheres nessa área (WERMELINGER M, et al., 2010). É notório que a divisão sexual do trabalho existe em todos os setores, relacionando determinadas atividades aos homens e vice-versa.

Do ponto de vista do papel da mulher, segundo Baylão AL e Schettino EM (2014), nos últimos cinquenta anos o contínuo crescimento da participação feminina é explicado por uma combinação de fatores econômicos e culturais. "Primeiro, o avanço da industrialização transformou a estrutura produtiva, a continuidade do processo de urbanização e a queda das taxas de fecundidade, proporcionando um aumento das possibilidades de as mulheres encontrarem postos de trabalho na sociedade".

Identificou-se, ainda, que todos os gestores das UBS de Natal não dispõem da formação específica para exercer o cargo de gestor em saúde, todos possuem alguma formação, mas não diretamente voltada para o cargo que exercem.

Destaca-se, todavia, que $75 \%$ dos gestores afirmaram ter feito algum curso de capacitação em gestão. Existem diferentes níveis de formação entre os gestores, desde pós-graduação, especialização e até alguma graduação. Dentre as graduações, encontra-se: administração, enfermagem, teologia, jornalismo, história.

Sobre isso, sabe-se que a formação de profissionais para atuar no âmbito da gestão em saúde é um desafio enfrentado diariamente nos serviços. Um profissional gestor que esteja preparado para liderar e planejar uma organização é de suma importância para que a instituição obtenha êxito na assistência em saúde no âmbito do SUS (BATISTA KBC e GONÇALVES OSJ, 2011).

Levando em consideração a complexidade operacional de uma organização de saúde, mudanças e transformações do cenário da saúde e a gestão de processos, a visão que um profissional formado na área da Gestão detém acerca de todas essas particularidades é completamente específica, importante e diferenciada em relação aos profissionais graduados em cursos que sejam da área da saúde ou não, pois as competências abordadas direcionam esses profissionais da Gestão nas necessidades diárias da organização (CHANES M, 2006).

O serviço de saúde possui infraestrutura de instalações, equipamentos, instrumentais, médicos, funcionários, recursos financeiros e especialmente os pacientes que ali se dirigem em busca de tratamento ou consulta. Para administrar adequadamente tais recursos, faz-se necessário um gestor competente, capaz de entender de pessoas, dinheiro, tecnologia e dos processos necessários (LINO KM, et al., 2008).

Ao se considerar a atividade de gestão como o princípio norteador dos serviços de saúde, se demonstra importante identificar os possíveis fatores geradores das incoerências e insatisfações destes serviços. Dependendo das condições de trabalho que o profissional da saúde conviva, pode desenvolver insatisfação e situações estressantes, isso vale para os mais diversos setores que os profissionais estejam inseridos.

Linch GF, et al. (2010) afirmam que "o ambiente hospitalar pode constituir um importante estressor para os profissionais devido aos possíveis sofrimentos vivenciados nesse local, às condições de trabalho, às demandas requeridas pela assistência, e também pela grande responsabilidade exigida no trabalho". Aspecto que pode explicar o alto nível de gestores com estresse identificados pela pesquisa.

Posto isso, vale salientar que a ausência de produção científica direcionada ao tema do presente artigo estresse em gestores em saúde - dificulta a elaboração de comparações e verificações do assunto abordado. 
No entanto, será discutida a partir de estudos de estresse em outros grupos pertencentes à área da saúde, uma vez que se trata do mesmo ambiente de trabalho. Para tanto, inicialmente vale reafirmar a importância do desenvolvimento de conteúdo científico quanto ao estresse ocupacional de gestores, para que possam ser vislumbradas estratégias de enfrentamento para essa.

Dos gestores entrevistados, $29(65,9 \%)$ apresentaram estresse, valor que mostra um diagnóstico preocupante, pois a sobrecarga gera a diminuição da produtividade e do desempenho, refletindo diretamente nos resultados das unidades de saúde em termos de qualidade, comprometendo a saúde do trabalhador e dos usuários, uma vez que necessitam dos serviços prestados por elas.

Com isso, o desenvolvimento do estresse por parte de um profissional passa a ser um elemento capaz de gerar desordens no funcionamento das organizações e serviços de um modo geral (CAMPOS EP, et al., 2010; ESTEVES GGL, et al., 2019).

Nesse contexto, sabe-se que os profissionais de saúde enfrentam situações conflituosas como controle supervisionado, excesso de trabalho e acúmulo de tarefas e isso pode causar desgaste físico e mental do profissional, comprometendo a sua saúde, como reforçam Camelo SH e Angerami EL (2004): "observa-se que são delegadas aos profissionais múltiplas tarefas com alto grau de exigência e responsabilidade, as quais, dependendo do ambiente, da organização do trabalho e do preparo para exercer seu papel, podem criar tensão para si, equipe e a comunidade assistida".

Sobre isso, houve destaque na amostra da fase de resistência, que é onde o indivíduo busca um equilíbrio, tentando manter a homeostase interna, a qual se apresentou com a maior prevalência no estudo 17 (38,6\%), seguido pela fase de exaustão, com $10(22,7 \%)$ gestores.

Faz-se importante destacar esse resultado se mostra preocupante, pois é na fase de exaustão que o sujeito não consegue manter-se físico e psicologicamente bem para realizar suas atividades diárias, abrindo portas para doenças mais graves aparecerem e em consonância com isso, diminuindo qualidade de vida do profissional que está diretamente ligada com a satisfação em exercer suas atividades na organização (LIPP ME, 2004).

Além disso, no estudo realizado os sintomas físicos predominaram (34; $77,3 \%)$, sendo um componente que já denota adoecimento, em que as consequências geradas desenvolvem prejuízos tanto para 0 trabalhador, quanto para a organização. Em contrapartida, existem medidas de enfrentamento, visando não só o bem-estar do trabalhador, mas também o melhor funcionamento dos serviços e assistência aos usuários (CAMPOS EP, et al., 2010).

Sobre isso, um renomado psicólogo americano fala sobre a importância da inteligência emocional, que é dividida em quatro pilares: autoconsciência, que ajuda a perceber quando você está se tornando estressado; autogestão, torna-o mais capaz de se acalmar; empatia, capacidade de entender o próximo e habilidades sociais, promovem a forma como você se expressa. Para ele, essas razões ajudam a lidar melhor com a vida (GOLEMAN D, 2012).

Além disso, para ajudar a superar uma crise, existem terapias, psicólogos, hábitos de vida, entre eles: dormir bem, usar técnicas de relaxamento, alimentação saudável, prática de atividades físicas, planejamento. Como também, a Estratégia de Coping, que é utilizada para que as pessoas se adaptem as circunstâncias adversas (CHAVES EC, et al., 2000).

Coping é definido como um conjunto de esforços, cognitivos e comportamentais, utilizado pelos indivíduos com o objetivo de lidar com demandas específicas, internas ou externas, que surgem em situações de stress e são avaliadas como sobrecarregando ou excedendo seus recursos pessoais (FOLKMAN S, LAZARUS RS, 1984).

Existe também uma discussão acerca da Qualidade de Vida no Trabalho (QVT), que é o conjunto das ações de uma organização que envolvem a implantação de melhorias e inovações gerenciais tecnológicas no ambiente de trabalho. A construção da qualidade de vida no trabalho começa a partir do momento que se olha a organização e as pessoas como um todo (LIMONGI-FRANÇA AC, 2008). 
Com isso, é possível afirmar que o profissional está exposto a fatores estressores diante de todas as responsabilidades assumidas, pressão e cobranças, portanto, é imprescindível que a organização possa compreender o quão importante a QVT é para o trabalhador e para o serviço, visando sempre elevar a qualidade de vida e, a partir disso, alavancar a satisfação no exercer das tarefas diárias (CHAVES EC, et al., 2000).

Levantando os dados colhidos no estudo, é possível afirmar que os trabalhadores que possuíam estresse na amostra eram mais jovens e atuavam como gestores há menos tempo, em contrapartida, estavam na unidade (exercendo outro cargo) há mais tempo.

É notório então, que na medida em que um profissional passa a desempenhar uma função que não era de sua competência, provoca possibilidade de gerenciamento desestruturado, em que a falta de conhecimento necessário para administrar gera situações conflituosas que desencadeiam o surgimento do estresse. Sobre isso, o artigo "Experiências dos enfermeiros com o trabalho de gestão em saúde no estado da Paraíba", publicado em 2010, retrata bem os desafios e despreparos dos enfermeiros na gestão em saúde, de modo que, apesar de terem conhecimento, mostram-se sensíveis no planejamento de algumas ações (BARRETO AJ, et al., 2010).

Analisando outros dados obtidos, foi possível identificar que a maior parte dos profissionais que possuía capacitação em gestão apontou possuir estresse, levantando a discussão que na medida em que o conhecimento cresce, as responsabilidades aumentam, sendo um fator para o desencadeamento de estresse. Entretanto, é indispensável o conhecimento e habilidades de função gerencial, como também a visão de melhoria de qualidade de vida no trabalho, que pode trazer ferramentas para enfretamento do estresse ocupacional (CHANES M, 2006).

O estudo possui limitações diante da realidade de apenas um Município, literatura escassa sobre o tema e a dificuldade de agendamento com os Gestores, de modo que nem todos foram entrevistados. Além disso, o instrumento utilizado permitia a autorreferência de sinais e sintomas pelos gestores, não sendo, portanto, confiável do ponto de vista de diagnóstico do processo de adoecimento. Os resultados apresentados, assim, devem ser avaliados a partir de tais limitações.

Pode-se dizer que suscitar conhecimento e reflexões sobre a temática é uma característica importante, assim, o estudo em tela espera trazer inquietações diante da realidade estressante dos Gestores das Unidades Básicas, estimulando interesse para futuras pesquisas acerca desse tema, que ainda é escasso, além de buscar ferramentas que possam ajudar não só a melhoria de vida desses profissionais, mas em concordância com isso, o melhor serviço nas organizações de saúde.

\section{CONCLUSÃO}

Evidenciou-se que a maioria dos Gestores das Unidades Básicas de Saúde estudadas possui estresse, e uma parcela desses já se insere na fase de Exaustão, que é quando o indivíduo começa a ficar suscetível ao surgimento de doenças. Além disso, os resultados obtidos indicam que existiu relação significativa entre a presença de Capacitação em Gestão e a presença de estresse, fator que pode ser explicado ao refletir que o maior conhecimento gera maior responsabilidade, contribuindo assim para o surgimento de fatores estressores.

\section{REFERÊNCIAS}

1. ABREU KL, et al. Estresse ocupacional e Síndrome de Burnout no exercício profissional da psicologia. Psicologia: ciência e profissão, 2002; 22(2): 22-29.

2. BARRÊTO AJ, et al. Experiências dos enfermeiros com o trabalho de gestão em saúde no estado da Paraíba. Texto \& Contexto-Enfermagem, 2010; 19(2): 300-308.

3. BATISTA KBF, GONÇALVES OFJ. Formação dos Profissionais de Saúde para o SUS: significado e cuidado. Saúde e Sociedade. 2011; 20(4): 884-899.

4. BAYLÃO AL, SCHETTINO EM. A Inserção da Mulher no Mercado de Trabalho Brasileiro. 2014; In: XI Congresso de Excelência em Gestão e Tecnologia. 
5. CAMELO SH, ANGERAMI EL. Sintomas de estresse em trabalhadores de cinco núcleos de Saúde da Família. Revista Latino-Americana de Enfermagem, 2004; 12(1): 14-21.

6. CAMPOS EP, et al. Equipes do programa saúde da família: estresse profissional e dinâmica de trabalho. Revista de APS. 2010; 13(1): 46-54.

7. CHANES, M. Os desafios na formação de gestores líderes em saúde. O mundo da saúde. 2006; 30(2): 326-331.

8. CHAVES EC, et al. Coping: significados, interferência no processo saúde-doença e relevância para a enfermagem. Revista da Escola de Enfermagem da USP. 2000; 34(4): 370-375.

9. COUTINHO DA, SANTOS JW. O estresse no mundo do trabalho: Uma abordagem individual e organizacional. Revista Cientifica Eletrônica de Psicologia, 2010; 8(14): 1-9.

10. ESTEVES GGL, et al., Fadiga e Estresse como preditores do Burnout em Profissionais da Saúde. Revista Psicologia: Organizações e Trabalho. 2019; 19(3): 695-702.

11. FOLKMAN S, LAZARUS RS. Stress, appraisal, and coping. New York: Springer Publishing Company; 1984. 60p.

12. GEREMIAS LM, et al. Prevalência do diabetes mellitus associado ao estresse ocupacional em trabalhadores bancários, Minas Gerais, Brasil. Revista CUIDARTE, 2017; (8)3: 1863-1874.

13. GOLEMAN D. A Inteligência Emocional. Rio de Janeiro: Objetiva, 2012; 384p.

14. LENTINE EC, et al. Estresse de profissionais de saúde das Unidades Básicas do município de Londrina. Revista Terra e Cultura, 2003; (19)37: 103-123.

15. LIMONGI-FRANÇA AC. Qualidade de Vida no Trabalho - QVT: conceitos e práticas nas empresas da sociedade pósindustrial. São Paulo: Atlas, 2004; 224p.

16. LINCH GF, et al. Estresse e profissionais da saúde: produção do conhecimento no centro de ensino e pesquisas em enfermagem. Cogitare Enfermagem, 2010; (15)3: 542-547.

17. LINO KM, et al. Administração hospitalar por competência: o administrador como gestor hospitalar. Trabalho de Conclusão de Curso (Curso de Administração) - Escola Superior de Amazônia, Belém, 2008; 29p.

18. LIPP ME. Stress no Brasil: Pesquisas avançadas. Campinas: Papirus, 2004; 223p.

19. LIPP ME. Stress: conceitos básicos. In: LIPP ME. Pesquisa sobre estresse no Brasil: saúde, ocupações e grupos de risco. Campinas: Papirus, 1996; 304p.

20. MAFFIA LN, PEREIRA LZ. Estresse no trabalho: estudo com gestores públicos do estado de Minas Gerais. Revista Eletrônica de Administração. 2014; 79(3): 658-680.

21. STARFIELD B. Atenção Primária: equilíbrio entre a necessidade de saúde, serviços e tecnologias. Brasília: UNESCO. Ministério da Saúde; 2002. 177p.

22. WERMELINGER M, et al. A Força de trabalho do setor de saúde no Brasil: focalizando a feminização. Revista Divulgação em Saúde para Debate. 2010; 45: 55-71. 